The study was consistent with an intraventricular tumor rather than a hematoma. The MRI revealed a large irregular lobulated mass believed to be a tumor arising from the corpus callosum, possibly a lipoma. The CT revealed a nonenhancing, hyperdense mass in the left frontal horn interpreted as an intraventricular hemorrhage. A CT-guided needle biopsy of the mass showed gemistocytic cells resembling large reactive astrocytes. A cardiac echocardiogram revealed multiple nodules in the intraventricular septum, consistent with multiple rhabdonyomas. Funduscopic exam revealed two small retinal hamartomas in the right eye. Ultrasound of the kidneys was negative. At eight weeks of age the infant had developed multiple hypopigmented macules on trunk and extremities. (Hahn JS et al. Neonatal subependymal giant cell astrocytoma associated with tuberous sclerosis: MRI, CT, and ultrasound correlation. Neurology Jan 1991; 41:124-128).

COMMENT. The interpretation of the echoencephalogram was closer to the correct diagnosis than that of the MRI and CT. Even without the needle biopsy of the mass the diagnosis of tuberous sclerosis and associated tumor may have been suspected and almost certainly determined by the echocardiogram and cardiac rhabdomyomas. Echoencephalogram may be the diagnostic study of choice in newborns. The MRI picture is atypical showing decreased signal intensity on T2-weighted imaging and increased signal intensity on T1-weighted studies.

\title{
SPASTIC DIPLEGIA AND THE MRI
}

The MRI findings in 34 children with spastic diplegia examined between 2 and 10 years of age are reported from the Seirei-Hamamatsu General Hospital, Mikatabara, Hammatsu, Shi zuoka; Nagoya City Medical School; and the National Rehabilitation Center for Disabled Children, Tokyo, Japan. There was dilation of the trigone, atrophy of the peritrigonal white matter, and prominent deep cortical sulci. The appearance of cerebral white matter was classified into four patterns: 1) normal; 2) peritrigonal white matter showing a thin contiguous line in one hemisphere; 3 ) peritrigonal white matter reduced in both hemispheres; and 4) the reduction of the white matter expanded anteriorly from the trigones to the bodies of the lateral ventricles. The degree of cerebral white matter loss correlated with severity of motor disability but not with severity of mental impairment. The distribution of periventricular high intensity T2-weighted images did not correlate with motor or mental disability. (Yokochi $\mathrm{K}$ et al. Magnetic resonance imaging in children with spastic diplegia: Correlation with the severity of their motor and mental abnormality. Dev Med Child Neurol Jan $1991 ; 33: 18-25)$.

COMMENT. The MRI findings were similar to those reported with periventricular leukomalacia. In infancy leukomalacia is characterized by delayed myelination and demonstrated as high intensity areas on T2 weighted images. It occurs most commonly in preterm infants and all but three of the children in this study had birth weights lower than 2000 grams. Mild degrees of periventricular white matter reduction are of ten interpreted as a normal variant in children, but correlations with delay in motor development should be considered. 